BMJ Open Sport \& Exercise Medicine
To cite: De Bosscher $\mathrm{R}$, Dausin C, Claus P, et al. Endurance exercise and the risk of cardiovascular pathology in men: a comparison between lifelong and late-onset endurance training and a nonathletic lifestyle - rationale and design of the Master@ Heart study, a prospective cohort trial. BMJ Open Sport \& Exercise Medicine 2021;7:e001048. doi:10.1136/ bmjsem-2021-001048

- Additional supplemental material is published online only. To view, please visit the journal online (http://dx.doi. org/10.1136/bmjsem-2021001048)

RDB and $C D$ are joint first authors.

Accepted 22 March 2021

Check for updates

(C) Author(s) (or their employer(s)) 2021. Re-use permitted under CC BY-NC. No commercial re-use. See rights and permissions. Published by BMJ.

For numbered affiliations see end of article.

Correspondence to Dr Ruben De Bosscher; ruben.debosscher@uzleuven.be

\title{
Endurance exercise and the risk of cardiovascular pathology in men: a comparison between lifelong and late- onset endurance training and a non- athletic lifestyle - rationale and design of the Master@Heart study, a prospective cohort trial
}

Ruben De Bosscher (D , ,,2 Christophe Dausin, ${ }^{3}$ Piet Claus, ${ }^{1}$ Jan Bogaert, ${ }^{4}$ Steven Dymarkowski, ${ }^{4}$ Kaatje Goetschalckx, ${ }^{2}$ Olivier Ghekiere, ${ }^{5}$ Ann Belmans, ${ }^{6}$ Caroline M Van De Heyning, ${ }^{7}$ Paul Van Herck, ${ }^{7}$ Bernard Paelinck, ${ }^{7}$ Haroun El Addouli, ${ }^{7}$ André La Gerche, ${ }^{8}$ Lieven Herbots, ${ }^{9}$ Hein Heidbuchel, ${ }^{7}$ Rik Willems, ${ }^{1,2}$ Guido Claessen ${ }^{1,2}$

\section{ABSTRACT}

Introduction Low and moderate endurance exercise is associated with better control of cardiovascular risk factors, a decreased risk of coronary artery disease and atrial fibrillation (AF). There is, however, a growing proportion of individuals regularly performing strenuous and prolonged endurance exercise in which the health benefits have been challenged. Higher doses of endurance exercise have been associated with a greater coronary atherosclerotic plaque burden, risk of AF and myocardial fibrosis (MF).

Methods and analysis Master@Heart is a multicentre prospective cohort study aiming to assess the incidence of coronary atherosclerosis, $\mathrm{AF}$ and $\mathrm{MF}$ in lifelong endurance athletes compared to late-onset endurance athletes (initiation of regular endurance exercise after the age of 30 years) and healthy non-athletes.

The primary endpoint is the incidence of mixed coronary plaques. Secondary endpoints include coronary calcium scores, coronary stenosis $>50 \%$, the prevalence of calcified and soft plaques and AF and MF presence. Tertiary endpoints include ventricular arrhythmias, left and right ventricular function at rest and during exercise, arterial stiffness and carotid artery intima media thickness. Two hundred male lifelong athletes, 200 late-onset athletes and 200 healthy non-athletes aged $45-70$ will undergo comprehensive cardiovascular phenotyping using $\mathrm{CT}$, coronary angiography, echocardiography, cardiac MRI, 12-lead ECG, exercise ECG and 24-hour Holter monitoring at baseline. Follow-up will include online tracking of sports activities, telephone calls to assess clinical events and a 7-day ECG recording after 1 year.

Ethics and dissemination Local ethics committees approved the Master@Heart study. The trial was launched on 18 0ctober 2018, recruitment is complete and inclusions are ongoing.
Trial registration number NCT03711539

\section{INTRODUCTION}

Cardiovascular disease remains the most common cause of death worldwide, with an estimate of 17.8 million deaths in 2017 , accounting for $31.8 \%$ of all-cause mortality. There is comprehensive evidence supporting the health benefits of regular physical activity. From a cardiovascular perspective, regular exercise has been associated with a lower prevalence and better control of cardiovascular risk factors (CVRF) such as arterial hypertension, dyslipidaemia, obesity and diabetes. $^{2-5}$ As such, it is not surprising that coronary artery calcium (CAC) scores on CT coronary angiography (CTCA), the risk of developing coronary heart disease as well as all-cause and cardiovascular mortality are lower in individuals engaged in domestic work, leisure-time physical activity, walking and cycling. ${ }^{6-9}$ Furthermore, in the presence of atherosclerosis, higher exercise tolerance was associated with lower cardiovascular event rates and all-cause mortality. ${ }^{10}{ }^{11}$ In 2018 , Radford et al demonstrated that higher levels of cardiorespiratory fitness attenuate the risk of cardiovascular disease and that the amount of attenuation increases as the coronary atherosclerotic burden increases. ${ }^{12}$ Regular physical activity is therefore proposed as one 
of the most effective preventive lifestyle interventions with $150 \mathrm{~min}$ of moderate-intensity aerobic training per week reducing adverse events from coronary artery disease $(\mathrm{CAD})$ by $50 \%{ }^{13} 14$

In the past decades, an increasing number of middleaged and older individuals have been exceeding these recommendations, engaging in intense exercise and participating in mass endurance events. ${ }^{15} 16$ Currently, there is controversy about the prevalence of coronary artery disease in the higher end of the spectrum of physical activity. In 2008, Möhlenkamp et al reported higher CAC scores in marathon runners compared with riskfactor matched controls. ${ }^{17}$ The hypothesis of causality between prolonged high-intensity endurance exercise and coronary atherosclerosis was, however, limited by the high prevalence $(52 \%)$ of former smokers in marathon runners and the unknown history of other risk factors. Nevertheless, recent studies have shown similar findings. In a group of middle-aged men engaged in recreational or competitive endurance exercise, Aengevaeren et al reported higher CAC scores and a higher prevalence of coronary plaques in participants with the highest lifelong exercise volume $(>2000$ metabolic equivalent (MET)-min/week) and with the highest dose of very vigorous exercise ( $\geq 9 \mathrm{MET}) .{ }^{18}$ However, many participants were current $(4.9 \%)$ and former $(38 \%)$ smokers and had a family history of coronary heart disease $(31.3 \%)$. The latter risk factors could have influenced lifestyle changes, leading to a compensatory higher exercise volume in those carrying a higher cardiovascular risk history. In a study by Merghani et al, master athletes, compared with non-athletic controls with an equally low Framingham risk score, were more likely to have coronary plaques and elevated CAC scores. ${ }^{19}$ These two last studies further emphasised a significant difference in plaque composition, with athletes having more calcified, less mixed and similar proportion of non-calcified plaques. These findings are relevant as calcified plaques are proven more stable. In contrast, non-calcified and particularly mixed plaques are more vulnerable and prone to rupture, hence carry a higher risk of major adverse cardiovascular events. ${ }^{20}$ This has recently been confirmed by DeFina $e t$ $a l$, who showed that higher levels of physical activity were associated with more prevalent CAC but without a significant increase in all-cause and cardiovascular disease mortality rates during 10.4 years follow-up. ${ }^{21}$

The differing degree of coronary artery disease, including plaque composition and outcome, in athletes suggests non-conventional pathophysiology. Cardiovascular risk factors, such as age, a current and past history of smoking and a history of dyslipidaemia, remain relevant. ${ }^{22}$ However, in cases of low cardiovascular risk profile, exercise-related hypertension, coronary spasms, disruption of laminar flow, inflammation and elevated parathyroid hormone (PTH) levels have been proposed as potential instigators of atherosclerosis. ${ }^{23-26}$ Perhaps a combination of all of the above is needed for coronary atherosclerosis to develop in physically active individuals.
Using CTCA, Lin et al evaluated coronary morphology before and after an extreme 140-day race in eight runners. ${ }^{27}$ Four runners with cardiovascular risk factors (smoking, hypertension) had coronary atherosclerosis at baseline, which progressed after the race. The remaining four runners were free from coronary atherosclerosis at baseline and did not develop any new coronary plaques post-race. Cardiovascular risk factors and coronary plaques might, therefore, be prerequisites for exercise to induce further atherosclerosis.

Similar to CAD, the relationship between exercise and atrial fibrillation (AF) is not straightforward. Concerning exercise, both the intensity and volume of exercise must be taken into account.

On the one hand, low to moderate-intensity exercise has been associated with a decreased risk of AF. In 2008 Mozaffarian et al reported a lower incidence of AF in individuals aged 65 years or older performing low to moderate physical activity such as walking. ${ }^{28}$ In the Troms $\varnothing$ study, investigators prospectively examined the association between physical activity and hospital-diagnosed AF in over 20000 adults and demonstrated a 19\% lower risk of $\mathrm{AF}$ in moderately active individuals. ${ }^{29}$

On the other hand, higher exercise volume increases the risk of AF. Several studies have reported a higher incidence of $\mathrm{AF}$ when endurance exercise was performed more frequently (ie, $>4$ /week) and longer (ie, $>5$ hours/ week) or when a lifetime history of $>2000$ hours was met. ${ }^{30-32}$ A 2009 meta-analysis calculated that endurance athletes were 5.3 times more likely to develop AF than controls. ${ }^{33}$ The importance of substantial lifetime exposure to high-intensity endurance exercise has also been illustrated in a 2018 paper by Opondo et al reporting that 10 months of dedicated high-intensity exercise training induces changes in left atrial structure and mechanical function but without electrical consequences in middleaged adults. ${ }^{34}$

Another potential exercise-related cardiac detriment is myocardial fibrosis (MF). A body of research has reported a heterogeneous profile of myocardial scarring in endurance athletes concerning prevalence, location, extent, aetiology and clinical significance.

In 2008, Möhlenkamp et al documented a higher than expected prevalence of MF, as defined by late gadolinium enhancement (LGE) on cardiac magnetic resonance (CMR) imaging, in ostensibly healthy marathon runners compared with age-matched control subjects $(12 \%$ vs $4 \%) .{ }^{17}$ In subsequent studies, the prevalence of MF has ranged between $0 \%$ to $50 \%$ of athletes. ${ }^{17}{ }^{35-38}$ Differing patterns and locations of myocardial scar have been described in athletes. A first distinction exists between a CAD pattern, defined as subendocardial or transmural scarring in a coronary perfusion area, and a non-CAD pattern of MF. ${ }^{1719} 35$ As examples of a non-CAD pattern, subepicardial and midmyocardial lateral wall fibrosis of the left ventricle $(\mathrm{LV})$ as well as MF at the interventricular septum and right ventricle (RV) insertion points have been reported. ${ }^{17353839}$ Based on these patterns, several 
aetiologies of MF have been proposed. A CAD pattern of fibrosis has been associated with an increasing CAC burden and significant coronary luminal stenosis. ${ }^{17} 19$ However, in some cases of CAD pattern of MF, no coronary abnormalities were found, and non-atherosclerotic mechanisms were suggested (ie, coronary spasm, increased thrombogenicity and coronary emboli). ${ }^{19}$ Subepicardial/midmyocardial lateral wall fibrosis might be caused by prior myocarditis and hinge point fibrosis by RV overload during exercise. The latter is supported by the association with cumulative exercise exposure, such as years of training and the lifetime number of races and race distances. ${ }^{35} 3638$

Most research was conducted in asymptomatic athletes meaning that MF in athletes was more often than not an incidental finding. Further research demonstrated that the clinical relevance of myocardial scarring varies and depends in part on its location. A CAD pattern and non-CAD subepicardial/midmyocardial MF have been associated with potentially life-threatening arrhythmias such as non-sustained ventricular tachycardia, but also ventricular tachycardia leading to sudden death or an appropriate shock by an implanted defibrillator. ${ }^{19} 39$ Postmortem evaluation in 357 cases of sudden death in athletes revealed a strong association between LV fibrosis and sudden cardiac death. ${ }^{40}$ Furthermore, large areas of subepicardial fibrosis of the LV have been linked with progressive LV dysfunction. ${ }^{41}$ By contrast, RV insertion point fibrosis is considered a benign finding, although it has been associated with a lower right ventricular ejection fraction (RVEF) at rest. ${ }^{38}$

The mentioned studies have focused on the detection of focal MF by use of LGE. In recent years native T1 mapping has emerged as a technique to measure extracellular volume, enabling diffuse fibrosis detection. Some studies comparing athletes to non-athletes have measured similar T1 and extracellular volume (ECV) values. ${ }^{36} 4243$ Others have reported lower T1 and higher ECV values in athletes with values remaining within normal ranges. ${ }^{44}$ In clinical practice, the use of T1-mapping is particularly useful to detect diffuse MF in cardiomyopathies such as hypertrophic cardiomyopathy and dilated cardiomyopathy. ${ }^{46}$

In conclusion, the health benefits of low and moderate intensity endurance exercise have been challenged at higher exercise loads. Carefully designed prospective studies using a multimodality approach in larger cohorts are needed to clarify the effect of prolonged and strenuous endurance exercise on the cardiovascular system. The Master@Heart study is a multicentre prospective cohort trial designed to assess the relationship between starting age of extensive endurance exercise, and as such lifetime dose, and the prevalence of coronary atherosclerosis, $\mathrm{AF}$ and $\mathrm{MF}$ in master athletes.

\section{METHODS AND ANALYSIS}

\section{Study design}

The Master@Heart (Master Athlete's Heart) study is a multicentre (University Hospitals Leuven, University Hospital Antwerp and Jessa Hospital Hasselt) prospective cohort study, funded by the Fund for Scientific Research, Flanders $(\mathrm{T} 003717 \mathrm{~N})$. The study schema is presented in figure 1 .

\section{Objectives and hypothesis}

The principal aim is to assess the prevalence of mixed, calcified and soft plaques in lifelong endurance athletes compared with endurance athletes who started training later in life and healthy non-athletic controls. The primary hypothesis is that lifelong endurance exercise, more than late-onset training, is associated with a lower prevalence of mixed plaques than non-athletic controls. As secondary aims, the Master@Heart study will assess the association between lifelong endurance training, MF and AF. The hypothesis is that prolonged exposure to endurance exercise is associated with a higher risk of $\mathrm{AF}$ and MF. The hypotheses are illustrated in figure 2.

\section{Study population and eligibility criteria}

An online screening questionnaire (www.masteratheart.be; online supplemental appendix) will be used to obtain information on gender, age, weight, length, current and previous smoking behaviour, load and timing of current and prior sports participation, CVRF, medication intake, previous cardiovascular conditions and family history in subjects willing to participate in the Master@Heart study (see online supplemental appendix). The questionnaire was built using REDCap electronic data capture tools hosted at the KU Leuven. ${ }^{4748}$ All subjects from this screening cohort will be evaluated for eligibility according to the criteria listed in box 1 .

Only male individuals will be eligible for inclusion in the Master@Heart trial because of the higher lifetime risk of coronary heart disease and $\mathrm{AF}$ in men relative to women. ${ }^{49} 50$ Moreover, as the risk of developing coronary heart disease before the age of 40 is low, we opted to include men between 45 and 70 years old. ${ }^{49}$ To identify an athlete through our online questionnaire, we will use a definition based on the hours per week of endurance training. For runners, we have put forward a cut-off of $\geq 6$ hours per week as a 2016 paper by Dawes and colleagues showed significantly more cardiac remodelling when performing $>5$ hours of exercise per week instead of $<5$ hours per week. ${ }^{51}$ Given the higher intensity of running than cycling, the inclusion criterion for cyclists is $\geq 8$ hours of cycling per week. Since cardiac volumes were similar between sedentary individuals and subjects performing $<3$ hours per week of exercise, we have chosen a cut-off of $<3$ hours per week of physical activity to define controls. ${ }^{51}$ 


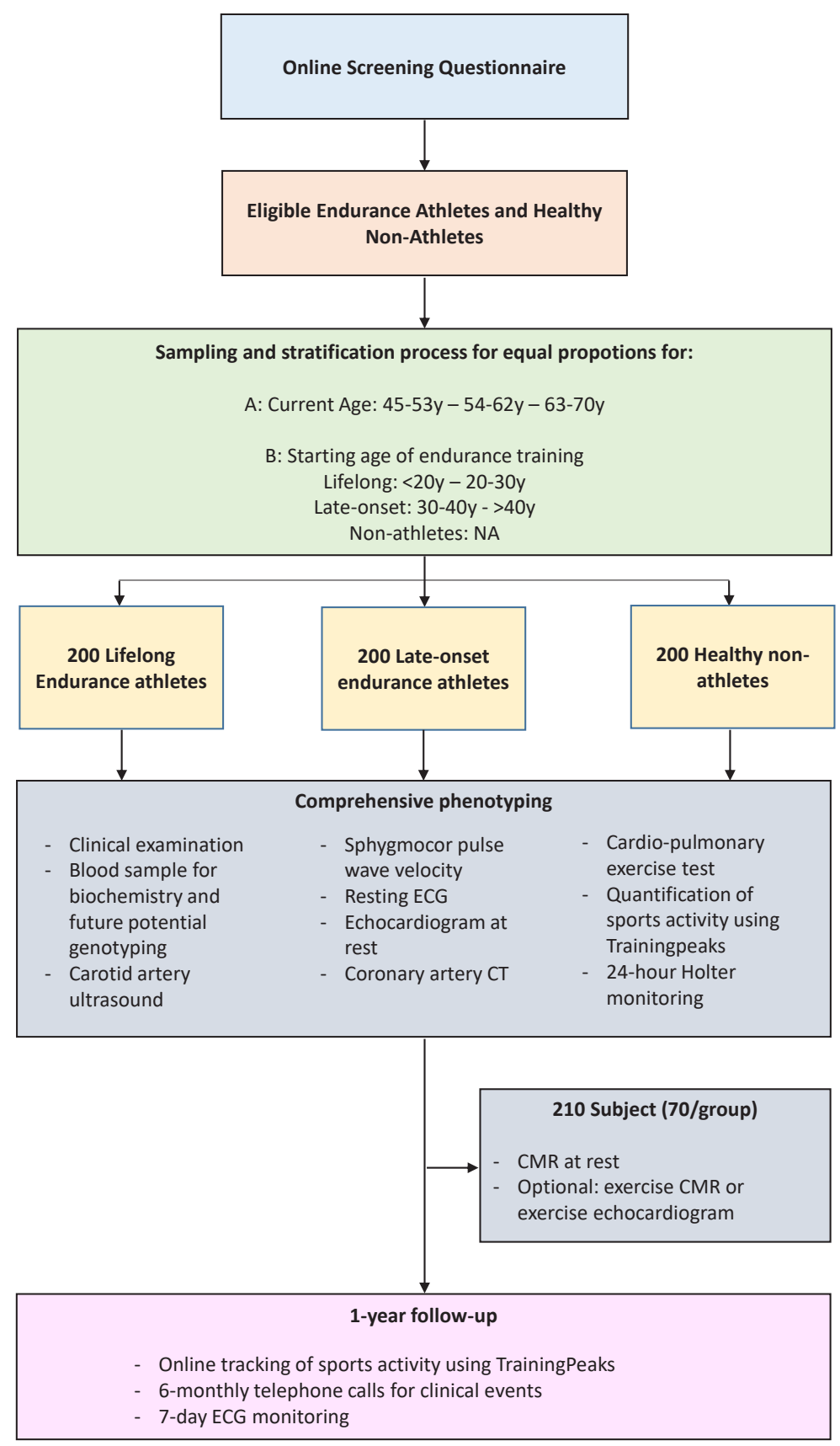

Figure 1 Study design and flow chart of the Master@Heart study. From an online screening questionnaire eligible individuals will be sampled and stratified in three groups (lifelong athletes, late-onset athletes, non-athletic controls) of 200 individuals. Baseline evaluation includes an overview of medical history, review of medication and supplements, physical examination, blood sampling, resting 12-lead ECG, two-dimensional and three-dimensional resting echocardiogram (TTE), carotid artery ultrasound, pulse wave velocity for arterial stiffness and non-invasive central blood pressure measurements using a Sphygmocor device, cardiopulmonary exercise testing including 12-lead exercise ECG and maximal oxygen consumption measurement, dual-energy X-ray absorptiometry, CT coronary angiography scan and a 24-hour Holter monitoring. Two hundred and ten subjects, 70 from each group, will undergo cardiac magnetic resonance (CMR) imaging. The latter subjects will also undergo cardiac imaging during exercise, using two-dimensional TTE (Antwerp and Hasselt) or CMR (Leuven). Follow-up consists of a 6-monthly telephone call for clinical events and a 7-day ECG monitoring at 1 year. y, years.

\section{Exclusion criteria}

To provide a clear view of the impact of intense endurance exercise on the cardiovascular system, we have decided to minimise the potential impact of CVRF by excluding subjects with known CVRF from the study (box 1). 


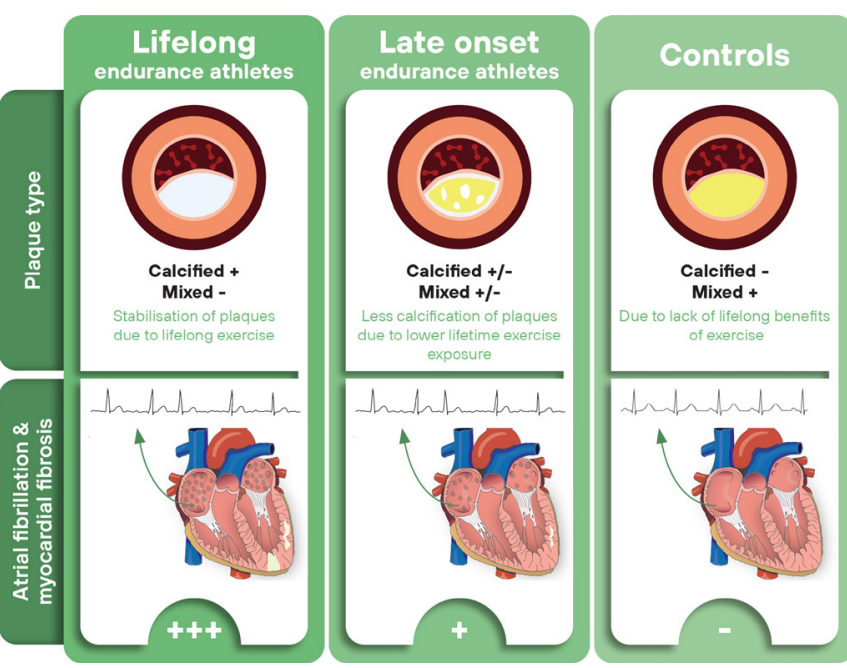

Figure 2 Study hypothesis of the Master@Heart study. The primary hypothesis is that lifelong endurance exercise, more than late-onset training, is associated with more calcified plaques and less mixed/and or soft plaques as compared with non-athletic controls. The secondary hypothesis is that prolonged exposure to endurance exercise is associated with a higher risk for atrial fibrillation and myocardial fibrosis.

Smoking, even in minimal doses, significantly increases the risk of coronary heart disease. ${ }^{52} 53$ The increased risk of atherosclerotic disease persists up to 30 years after smoking cessation. ${ }^{54}$ Additionally, concurrent or former smoking was a significant limitation in the interpretation of previous studies describing the association between exercise and coronary atherosclerosis. ${ }^{17} 18$ Therefore, current smoking and any smoking history is an exclusion criterion. A 2010 meta-analysis of nearly 700000 people reported a twofold increased risk of coronary heart disease associated with diabetes. ${ }^{55}$ Therefore, the use of any antidiabetic drugs is an exclusion criterion. With regard to lipids, elevated serum cholesterol levels are associated with death from coronary heart disease in middle-aged men during a 12-year follow-up. ${ }^{56}$ Additionally, statin use has

Box 1 Eligibility criteria for the Master@Heart study including both inclusion and exclusion criteria.

\section{Inclusion criteria}

1. Men aged $45-70$ years.

2. Athletes engaged in cycling $\geq 8$ hours per week or running $\geq 6$ hours per week or triathlon training (combination of swimming, cycling, running) $\geq 8$ hours per week for at least 6 months prior to baseline.

3. Non-athletes engaged in $\leq 3$ hours per week of physical activity without prior exposure to regular endurance exercise.

\section{Exclusion criteria}

1. Medical history of cardiovascular disease.

2. Current or past smoker.

3. Use of antidiabetic drugs.

4. Use of statins.

5. Use of antihypertensive drugs.

6. Body mass index $>27.2 \mathrm{~kg} / \mathrm{m}^{2}$

7. Allergy for iodine contrast agents. been associated with a higher prevalence of plaque calcification and greater progression of CAC without higher event rates, which has been interpreted as the ability of statins to modulate coronary plaques. ${ }^{57} 58$ This has been confirmed by serial intravascular ultrasound analysis showing that statin therapy was associated with plaque atheroma regression as well as the progression of plaque calcification. $^{59}$ Hence, individuals using statins will be excluded from our study. Elevated blood pressure has been related to an increase in cardiovascular events and mortality ${ }^{60}$ Individuals using antihypertensive drugs will, therefore, be excluded from the Master@Heart study. Finally, with regard to overweight and obesity, as a body mass index (BMI) between 24.26 and 27.21 did not confer a higher OR for CAC >10 Agatston units, we opted for a BMI cut-off of $>27.2 \mathrm{~kg} / \mathrm{m}^{2}$ as an exclusion criterion. ${ }^{61}$

From all eligible subjects based on the questionnaire, 600 will be sampled for inclusion. Sampling will be done randomly but stratified by current age (45-53 years, 54-62 years and 63-70 years) as well as age at which endurance training was started (Group 1-lifelong: subdivided in $<20$ years and 20-30 years, Group 2-lateonset: subdivided in 31-40 years and $>40$ years, Group 3-healthy non-athletes: NA). This will give equal proportions with regard to current age in all three groups and equal proportions with regard to starting age of endurance exercise in lifelong and late-onset athletes. As the prevalence of high-level endurance athletes is lower in older individuals, we opted for a participant distribution of three out of seven aged 45-53 years, three out of seven aged 54-62 years and one out of seven aged 63-70 years. The sampling and stratification strategy is illustrated in figure 3 .

\section{Study procedures}

Baseline evaluation will include an overview of medical history, review of medication and supplements, physical examination, blood sampling for biochemistry and genotyping, resting 12-lead ECG, two-dimensional and

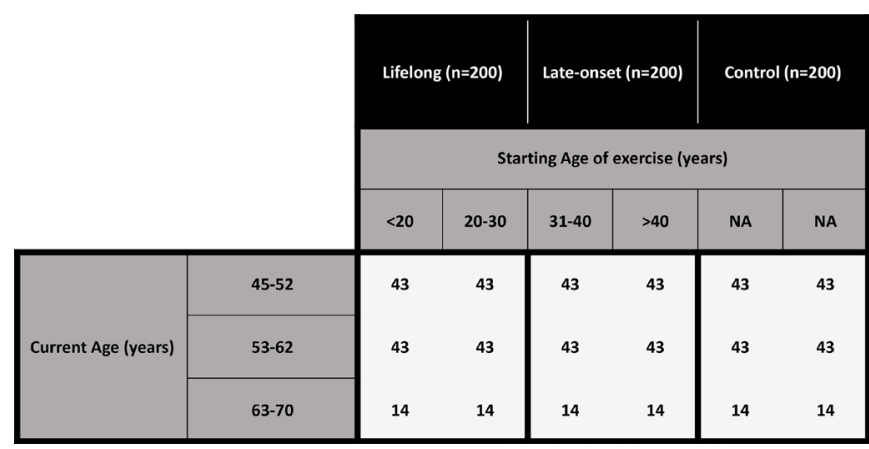

Figure 3 Sampling stratification of the Master@Heart study by current age (45-53 years, 54-62 years and 63-70 years) and age at which endurance training was started (lifelong: $<20$ years and 20-30 years; late-onset: 31-40 years and >40years; non-athletic controls: N/A). For current age a proportion of $3 / 7: 3 / 7: 1 / 7$ of individuals aged $45-53$ years, 54-62 years and 63-70 years, respectively, was applied. 
three-dimensional resting echocardiogram (TTE), carotid artery ultrasound, pulse wave velocity for arterial stiffness and non-invasive central blood pressure measurements using a Sphygmocor device, cardiopulmonary exercise testing (CPET) including 12-lead exercise ECG and maximal oxygen consumption measurement, dual-energy X-ray absorptiometry, CTCA scan and a 24-hour Holter monitoring in all 600 subjects. CMR imaging, including gadolinium contrast administration, will be performed in 210 randomly selected subjects, 70 from each group. Randomisation for CMR occurs at the initial sampling and stratification with a similar age distribution. This subgroup of study participants will also undergo cardiac imaging during exercise, using two-dimensional TTE (Antwerp and Hasselt) or CMR (Leuven). The sequence of investigations will differ between sites based on logistics.

To gain a broad cross-sectional view on the association between endurance exercise and cardiovascular pathology, subjects will be followed-up for a minimum of 1 year. Follow-up will consist of online tracking of sports activity using TrainingPeaks (Peaksware, Boulder, USA). At 6 months, a telephone call will assess clinical events such as alteration in medication, the onset of $\mathrm{AF}$, coronary interventions or major adverse cardiovascular events. Finally, at 1-year follow-up, a 7-day ECG-monitoring will identify $\mathrm{AF}$ and other arrhythmias.

A clinical report covering all relevant findings will be available to the patient and his general practitioner. For research purposes, all measurements and recorded events will be pseudonymised and stored in the REDCap database.

\section{Physical examination and blood samples}

The information derived from the physical examination and blood sampling, such as systolic and diastolic blood pressure, total, low-density lipoprotein (LDL) and highdensity lipoprotein (HDL) cholesterol, will be used to assess the individual cardiovascular risk score.

\section{2-lead ECG}

A resting 12-lead ECG will be recorded and interpreted following the Seattle Criteria by a cardiologist with experience in sports medicine. ${ }^{62}$

\section{Sphygmocor}

Carotid-femoral pulse wave velocity is a well-established marker of arterial stiffness and is linked to total cardiovascular risk. ${ }^{63}$ Using a SphygmoCor XCEL device (SphygmoCor device, AtCor Medical, Sydney, Australia), the central blood pressure, the central arterial pressure waveform, central aortic pressures and pulse wave velocities will be measured, allowing assessment of arterial stiffness. Pulse wave velocity is determined by simultaneous measurement of the carotid and femoral pulse by tonometry and volumetric displacement. The transit time between the feet of the two waves is calculated. Distances are measured from the suprasternal notch to the top of the thigh cuff, the site of carotid tonometry and the site where the femoral artery could be applanated by tonometry. $^{64}$

\section{Dual-X-ray absorptiometry}

Subjects will undergo a dual-energy X-ray absorptiometry scan (Discovery W, Hologic, Bedford, Massachusetts, USA - GE Lunar Prodigy Advance, GE Healthcare, Horten, Norway) to measure lean mass, fat mass, bone mineral content and bone mineral density of the whole body, trunk, legs and arms.

\section{Cardiopulmonary exercise testing}

Peak oxygen consumption (peak $\mathrm{VO}_{2}$ ) will be determined using a continuous bicycle stress test. After a 5 min warmup, resistance will gradually increase by $30 \mathrm{~W}$ every minute from an initial load of $60 \mathrm{~W}$ in cyclist and $30 \mathrm{~W}$ in runners and controls until exhaustion. ECG monitoring during the test will ensure analysis of heart rate, arrhythmias and repolarisation abnormalities. During the exercise test's final stages, the respiratory gas exchange will be analysed using a breath-by-breath open-circuit spirometry system. Peak $\mathrm{VO}_{2}$ will be determined as the highest $30 \mathrm{~s}$ average oxygen consumption. The first and second ventilatory threshold will be determined from respiratory gas analysis parameters.

\section{Two-dimensional and three-dimensional transthoracic echocardiography}

Two-dimensional and three-dimensional TTE will be performed using a Vivid E9 or E95 ultrasound system (GE Healthcare, Horten, Norway) with an active matrix single-crystal phased array transducer (GE M5Sc-D probe, GE Healthcare, Horten, Norway) and 1.5-4MHz matrixarray transducer (GE 4Vc-D Matrix 4D cardiac probe, GE Healthcare, Horten, Norway). Cardiac morphology will be assessed, including end-diastolic volume, end-systolic volume, rendering ejection fraction (EF) for both ventricles, as well as right and left atrial volumes. The diastolic function will be assessed using established Doppler and tissue-Doppler parameters such as the $\mathrm{E}$ wave velocity, the A wave velocity, the $\mathrm{E} / \mathrm{A}$ ratio, septal, lateral and averaged E', E/E', tricuspid regurgitation flow velocity and the S-D-A waves at the pulmonary veins. An in-depth analysis of the intrinsic myocardial function will be performed by strain analyses. RV and LV strain and strain rate will be assessed as systolic function measures. RV and LV early and late diastolic strain rate will be assessed for diastolic function. Time-to-peak shortening in all 18 segments of the LV and the RV free wall will be measured to assess for differences in timing and mechanical dispersion. Atrial strain analysis will be performed to assess the reservoir, conduit and contraction function of both atria. All measurements will be made following international guidelines. ${ }^{6566}$

\section{Carotid artery ultrasound}

The presence of atherosclerotic plaques at the common carotid artery and internal carotid artery will be evaluated 
using a ProSound Aloka Alpha 6 (Aloka Holding Europe AG, Zug, Switzerland), a Philips Epiq5 (Philips Medical Systems, Bothell, Washington, USA) or a Vivid E9 (GE Healthcare, Horten, Norway) ultrasound system with a UST-5413 (Aloka Holding Europe AG, Zug, Switzerland), L12-3 (Philips Medical Systems, Bothell, Washington, USA) or Vivid E9 (GE Healthcare, Horten, Norway) or a 9L (GE Healthcare, Horten, Norway) transducer. Pulse wave Doppler measurement will be used to assess the degree of stenosis when plaques are present. To further assess peripheral atherosclerotic burden, the intimamedia thickness will be measured as the distance between the hyperechogenic blood-intima line and the hypoechogenic media-adventitia line at the distal part $(1 \mathrm{~cm}$ proximal to the bulb) of the common carotid artery and the proximal part ( $1 \mathrm{~cm}$ distal to the bulb) of the internal carotid artery. All measurements will be made following international guidelines. ${ }^{6768}$

\section{CTCA}

CTCA will be acquired using a 128-slice dual-source CT scanner (Siemens Somatom Force-Siemens Healthineers, Forchheim, Germany) or a 256-slice CT scanner (GE Revolution-GE Healthcare, Milwaukee, Wisconsin) or a 320-slice CT scanner (Aquilion ONE ViSION_Canon Medical Systems, Otawara, Japan). To achieve a target heart rate of $<65$ beats per minute, the beta-blocker esmolol will be used intravenously when necessary. The choice for esmolol instead of the more conventionally used beta-blockers (ie, metoprolol) was based on esmolol's short half-life, which will prevent interference with other tests (ECG, CPET, TTE, CMR and Holter). All subjects will receive $0.4 \mathrm{mg}$ sublingual nitroglycerine $2 \mathrm{~min}$ before scanning. First, a nonenhanced ECG-synchronised scan will be taken for the quantification of coronary calcium rendering the CAC score. Second, an ECG-triggered CTCA will be acquired after the intravenous injection of iodinated contrast medium in an antecubital vein followed by a saline chaser. All coronary atherosclerotic lesions will be analysed and interpreted following the 2016 Society of Cardiovascular Computed Tomography (SCCT) guidelines on syngo.via (Siemens Healthineers, Forchheim, Germany) or GE Advanced Workstation (GE Healthcare, Milwaukee, Wisconsin) software with regard to their stenosis grade by the Coronary Artery Disease Reporting and Data System (CAD-RADS), composition (calcified, mixed, soft) as well as for their vulnerability (positive remodelling, low-attenuation plaque, spotty calcification and the napkin-ring sign). A density of $>130 \mathrm{HU}$ defines calcified areas. Calcified plaques are entirely composed of calcified areas. Mixed plaques are composed of both calcified and non-calcified areas. Soft plaques are entirely composed of non-calcified areas. The use of the syngo. via Frontier Coronary Plaque Analysis software (Siemens Healthineers, Forchheim, Germany) will further assess luminal and plaque volumes. ${ }^{69-71}$

\section{Cardiac MRI}

In 210 randomly selected participants (70 per group, matched for age), CMR will be performed using a 1.5T MRI scanner (Magnetom Aera 1.5T-Siemens Healthineers, Erlangen, Germany; Ingenia, Achieva or Ambition 1.5T-Philips Medical Systems, Best, The Netherlands), a dedicated cardiac coil and electrocardiographic gating. Steady-state free precision short-axis cine imaging ( $8 \mathrm{~mm}$ slice thickness without gaps) will be obtained to analyse cardiac mass, function and volumes. Also, native and post-contrast $\mathrm{T} 1$ mapping will be performed using the modified look-locker inversion recovery sequence to calculate ECV. MF will also be evaluated using delayed enhancement of breath-hold phase-sensitive inversion recovery sequences $10 \mathrm{~min}$ after administering gadolinium-diethylenetriamine pentaacetic acid. Analysis of all CMR data will be performed in a central core laboratory. Assessment of cardiac volumes and mass will be performed using CVI42 (Circle Cardiovascular Imaging, Calgary, Canada). IntelliSpace Portal (Philips Medical Systems, Eindhoven, The Netherlands) will be used for T1 and ECV mapping, whereas suiteHEART (NeoSoft, Pewaukee, USA) is used for strain analysis (feature tracking). Our validated robust nonrigid motion correction will be used for accurate $\mathrm{T} 1$ measurements and ECV calculations. ${ }^{72}$ T1-mapping will also provide an estimate of myocardial cellular mass as a feature of athletic remodelling. ${ }^{73}$ In University Hospitals, Leuven CMR imaging will also be performed during exercise. Our research group has previously demonstrated the feasibility, reliability and clinical utility of CMR to quantify cardiac volumes and function during exercise. ${ }^{74-76}$ The assessment of myocardial function during exercise will allow us to investigate whether the duration and intensity of long-term endurance exercise affect $L V$ and RV functional reserve.

\section{Exercise stress echocardiography}

In the University Hospital Antwerp and Jessa Hospital Hasselt, exercise stress echocardiography (Vivid E95 ultrasound system-GE Healthcare, Horten, Norway) will be performed instead of exercise CMR in the subjects having undergone CMR at rest. Measurements will be performed at rest and during several exercise stages depending on heart rate and respiratory gas analysis parameters. The following measurements and derived calculations will be collected: left ventricular ejection fraction (LVEF), RVEF, RV fractional area change, biventricular systolic and diastolic strain parameters, Doppler and tissue Doppler parameters to assess cardiac output, diastolic function and pulmonary artery systolic pressure. Using stress echocardiography, we will assess how prolonged high-intensity endurance training impacts pulmonary vascular resistance and diastolic function during exercise. All measurements will be made following international guidelines. ${ }^{77}$ 


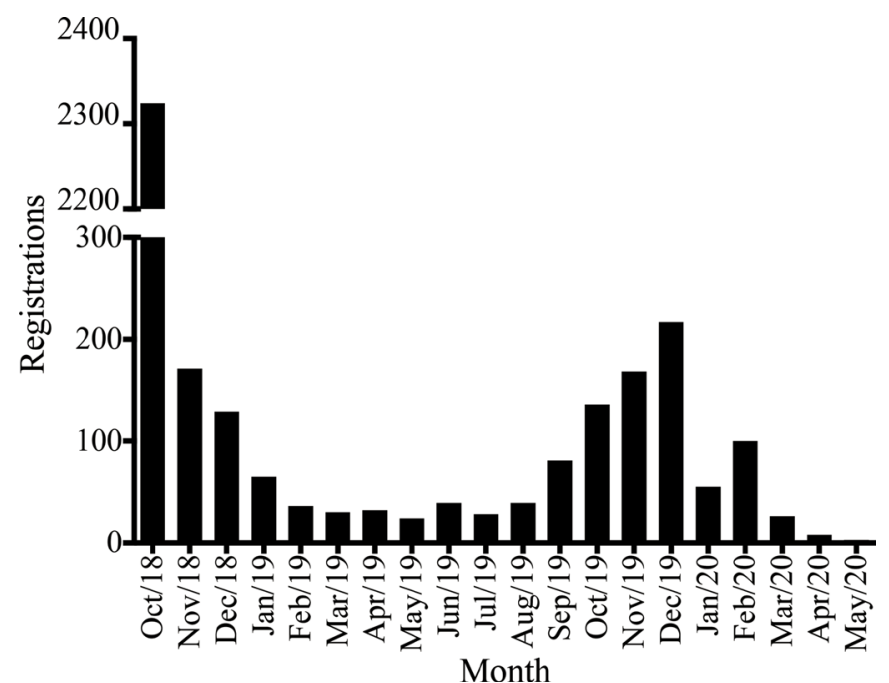

Figure 4 Bar graph of the registrations per month on the Master@Heart website.

\section{Holter analysis}

For the 24-hour Holter ECG monitoring, a Spiderview Holter device (ELA Medical, Paris, France) will be attached to BlueSensor VL ECG electrodes (Ambu, Penang, Malaysia). The ECG recordings will be analysed offline using SyneScope software (ELA Medical, Paris, France) to determine heart rate boundaries and to evaluate the occurrence of arrhythmias. Bradycardia is defined as a heart rate slower than 50 beats per min. A cardiac pause is defined as an interruption in the ventricular rate $>2 \mathrm{~s}$ and non-sustained ventricular tachycardia as three or more consecutive ventricular beats (origin below atrioventricular node) with an $\mathrm{RR}$ interval $<600 \mathrm{~ms}$ (ie, $>100$ beats per min) and lasting $<30$ s.

\section{Seven-day ECG monitoring}

For the 7-day ECG monitoring, a RootiRx (Rooti Labs, Taipei, Taiwan) will be used. RootiRx is an ECG patch monitoring device consisting of an integrated sensor system, a microelectronic board with memory storage and an internal rechargeable battery. RootiRx allows for continuous ECG monitoring for up to 7 days in $250 \mathrm{~Hz}$ frequencies with 24-bit high resolution. Recorded data is analysed by Rooti Labs developed algorithms and creates a report which is reviewed and edited by physicians and finally sent back to the referring physician. The final report includes the number of recording days, the amount of recorded beats, the average heart rate (overall, day and night), the maximum and minimum heart rate, the amount of cardiac pauses defined as an interruption in the ventricular rate $>2 \mathrm{~s}$. AF burden is reported as an amount of $\mathrm{AF}$ events, time in $\mathrm{AF}$ and percentage time in AF. Reported atrial events include atrial ectopic beats and supraventricular tachycardia, and ventricular events include ventricular ectopic beats, doublets, triplets, bigeminy, trigeminy and ventricular tachycardia. The performance of RootiRx has been validated against

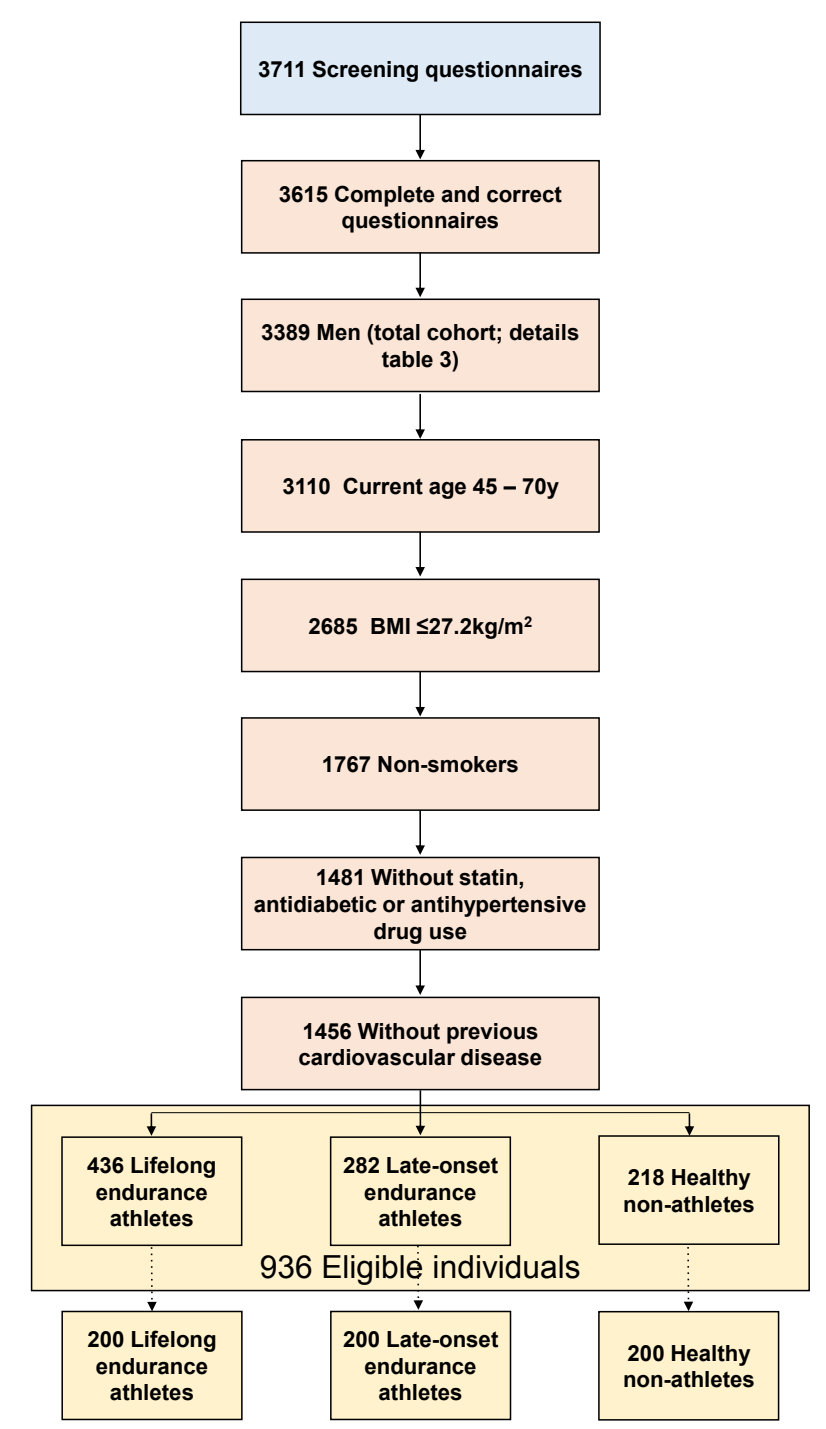

Figure 5 Flow chart of cohort selection. Step-by-step flowchart representing the inclusion of the three cohorts. Numbers represent the remaining eligible participants after application of the inclusion and exclusion criteria. BMI, body mass index, $y$, years.

standard 24-hour Holter monitoring in healthy individuals and patients with arrhythmias. ${ }^{78}$

\section{Endpoints}

The Master@Heart study's primary endpoint is the difference in the prevalence of mixed plaques in lifelong endurance athletes, late-onset endurance athletes and non-athletic controls. The main secondary endpoints are (1) prevalence of AF on 12-lead ECG, a 24-hour Holter monitoring or a 7-day ECG-monitoring, (2) the presence and quantification of MF as assessed by LGE imaging (\% of LV mass) and T1-mapping (\% of ECV) and (3) total CAC scores and the presence of $>50 \%$ stenosis in proximal coronary segments. Tertiary endpoints include: (1) prevalence of ventricular ectopic beats, non-sustained 

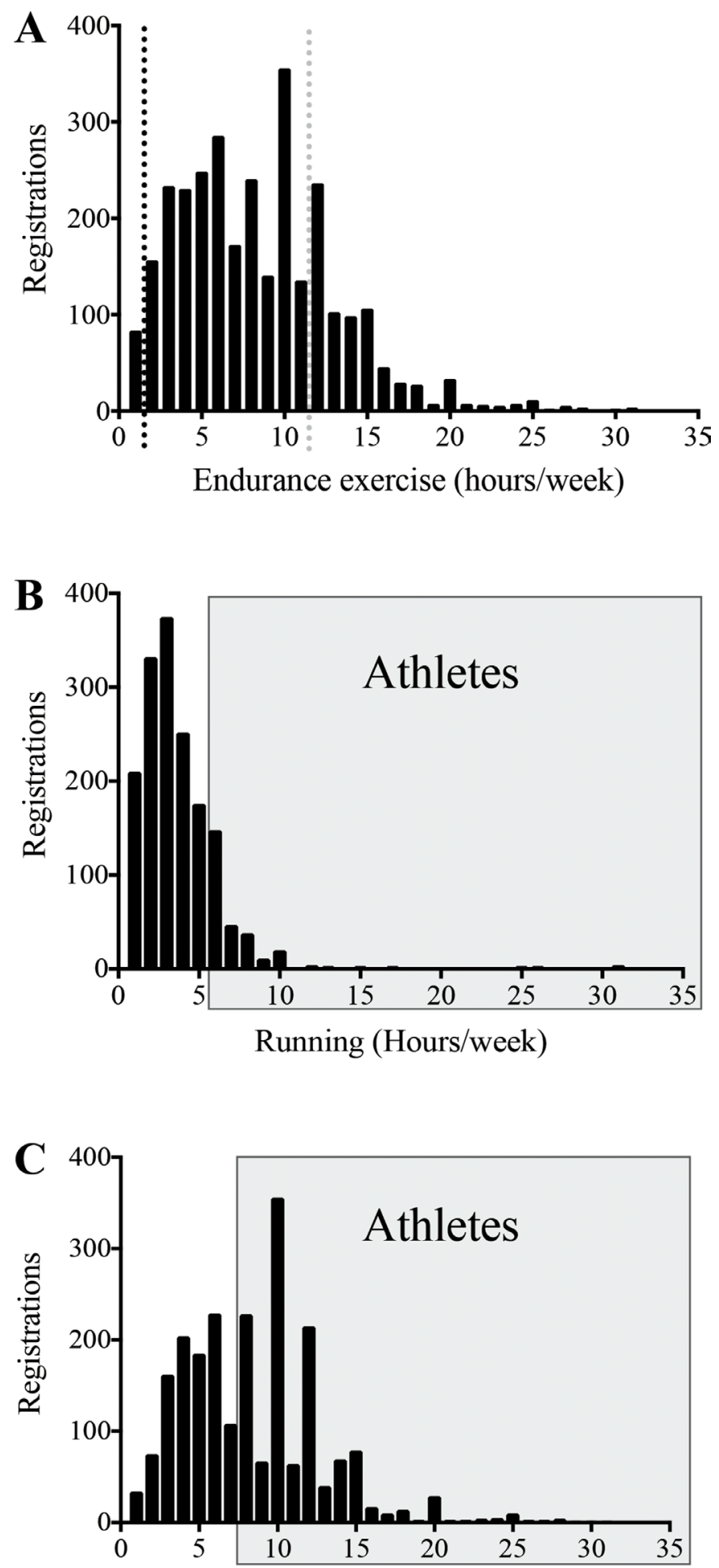

Cycling (Hours/week)

Figure 6 Distribution of endurance, cycling and running training hours. Panel $(A)$ depicts the distribution of endurance exercise hours per participant who filled in the screening questionnaire including the average training hours for controls and athletes as per the inclusion criteria. Panels (B) and $(C)$ show the distribution of running hours per week $(B)$ and cycling hours per week (C) of participants who filled in the screening questionnaire. The black frames indicate the participants that fulfil the criteria for athletes as per the study inclusion criteria.
Table 1 Demographic and physical activity data on eligible lifelong, late-onset athletes and healthy non-athletes

\begin{tabular}{llll}
\hline & $\begin{array}{l}\text { Non- } \\
\text { athletes } \\
(\mathbf{n = 2 1 8})\end{array}$ & $\begin{array}{l}\text { Lifelong } \\
(\mathbf{n = 4 3 6 )}\end{array}$ & $\begin{array}{l}\text { Late- } \\
\text { onset } \\
(\mathbf{n = 2 8 2})\end{array}$ \\
\hline Age (years) & $54.2 \pm 6.5$ & $53.5 \pm 5.9$ & $54.96 \pm 6.3$ \\
\hline Weight (kg) & $76.0 \pm 8.3$ & $74.63 \pm 7.4$ & $75.3 \pm 8.1$ \\
\hline $\begin{array}{l}\text { Length (cm) } \\
\text { Body mass index } \\
\text { (kg/m }{ }^{2} \text { ) }\end{array}$ & $178.3 \pm 6.5$ & $179 \pm 6.1$ & $179.3 \pm 6.2$ \\
\hline $\begin{array}{l}\text { Endurance exercise } \\
\text { (hours/week) }\end{array}$ & $1.5 \pm 1.3$ & $11.6 \pm 3.8^{*}$ & $11.3 \pm 4.2^{*}$ \\
\hline $\begin{array}{l}\text { Total physical activity } \\
\text { (hours/week) }\end{array}$ & $1.6 \pm 1.3$ & $11.8 \pm 3.9^{*}$ & $11.4 \pm 4.2^{*}$ \\
\hline $\begin{array}{l}\text { Start age endurance } \\
\text { exercise (years) }\end{array}$ & NA & $17.6 \pm 6.6$ & $40.7 \pm 6.4 \dagger$ \\
\hline
\end{tabular}

Demographic and physical activity data, based upon the screening questionnaire. Values are mean \pm SD.

${ }^{*} \mathrm{P}<0.05$. Unpaired analysis of variance comparison, significantly different from healthy non-athletes.

$\dagger p<0.05$. Unpaired comparison, significantly different from lifelong athletes.

and sustained ventricular tachycardia, a 24-hour Holter monitoring or a 7-day ECG monitoring; (2) LV and RV systolic function at rest and during exercise; (3) LV diastolic function by two-dimensional echocardiography including speckle tracking imaging; (4) bi-atrial function by two-dimensional echocardiography including speckle tracking imaging; (5) filling pressures and pulmonary artery pressures during exercise assessed by exercise echocardiography; (6) arterial stiffness by Sphygmocor; and (7) carotid intima media thickness as assessed by carotid artery ultrasound.

\section{STATISTICAL CONSIDERATIONS}

\section{Sample size and power calculations}

The Master@Heart study is powered to assess the association between the number of years (exercise age) middle-aged men have performed endurance exercise and the probability of mixed coronary plaques. The sample size calculations are based on the likelihood ratio $\chi^{2}$ test. Exercise age was assumed to be uniformly distributed between 0 and 40 years. A univariate logistic regression assessed the association between exercise age and the probability of mixed plaques. It was assumed that exercise can be assessed in the logistic regression as a linear, untransformed variable, that is, $\log _{2} f_{0}(\mathrm{p} /$ $(1-p))=\alpha+\beta \times$ exercise age. Statistical testing is two-sided and assessed at a significance level of $5 \%$. PROC POWER in SAS V.9.4 (SAS/STAT V.14.1) was used to calculate the sample size for a univariate logistic regression with exercise age as the only covariate. In line with recent research, the anticipated incidence of mixed plaques is $35 \%$ in subjects with an exercise age of 0 years ( $\mathrm{P} 0)$ and $22 \%$ in subjects with an exercise age of 25 years (P25). ${ }^{1819}$ 
Under these assumptions, a sample size of 486 subjects would offer a statistical power of $80 \%$ with a $5 \%$ probability of a type I error. In the Master@Heart study, 600 subjects (200 per group) will be included, which will give a margin of up to $20 \%$ uninterpretable data.

Analysis of variance and non-parametric approaches will be used to analyse continuous outcomes. For dichotomous outcomes, $\chi^{2}$ tests will be used. The relationship between calcium score and current and historic exercise load will be assessed using Pearson or Spearman correlation as appropriate. Multiple linear, non-linear and logistic regression analysis will be used to determine the association between coronary atherosclerosis or AF and endurance exercise load and cardiovascular risk factors. Losses to follow-up are expected to be minimal as extensive phenotyping is performed at baseline. A subsequent follow-up will consist of telephone calls and a single 7-day heart rate monitoring.

\section{TRIAL STATUS}

The Master@Heart study was officially launched on 18 October 2018 with a press event organised in collaboration with Golazo (Paal-Beringen, Belgium). Golazo is an organiser of sporting events in Belgium and promotes all aspects of physical activity. On the same day, our website and online registration tool were launched (www. masteratheart.be). Potential candidates could register and received a personal code that provided access to an online questionnaire linked to the REDCap database (online supplemental appendix 1). The information from the questionnaire was pseudonymised. The launch resulted in a peak of registrations in the first month, after which a decline in registrations was observed until July 2019 (figure 4). Therefore, in the second half of 2019, a social media campaign was set up in combination with the distribution of flyers at several national sporting events, which led to an increase of registrations until January 2020.

As of May 26 2020, 3711 registrations were obtained, of which 3615 (97\%; 3389 men) were completed correctly (figure 5). Out of these 3389 male participants, 1933 were excluded based on the exclusion criteria specified in box 1. As depicted in figure 6, 936 of the remaining 1456 participants based on the questionnaire (online supplemental appendix) fulfilled the inclusion criteria for physical activity. These eligible participants will be sampled after stratification by (1) age at which endurance training was started and (2) current age. Non-athletes had a slightly higher BMI $\left(23.9 \pm 1.9\right.$ vs $23.3 \pm 1.7 \mathrm{~kg} / \mathrm{m}^{2}$, $\mathrm{p}<0.05$, table 1) than lifelong athletes. No anthropometrical differences were observed between lifelong and late-onset athletes. Per inclusion criteria, the amount of endurance exercise per week in non-athletes was lower than in late-onset and lifelong athletes $(1.5 \pm 1.3 \mathrm{vs}$ $11.3 \pm 4.2$ vs $11.6 \pm 3.8$ hours/week, respectively; $\mathrm{p}<0.05$ ).

The first participant gave informed consent and took part in the study on 21 February 2019, and recruitment is expected to finish in 2021 with a delay of 6-12 months due to the COVID-19 pandemic. Trial results will be communicated through publications. Published articles will be available to the community on www.masteratheart. be.

\section{CONCLUSION}

A better understanding of the relationship between intense and prolonged endurance exercise and atherosclerosis, MF, arrhythmias and other cardiovascular diseases in middle-aged and older athletes is needed. Athletes have increased longevity but are not granted immunity from cardiovascular disease. Whether or not there is a threshold at which the risks of endurance training outweigh the benefits remains unanswered.

The rigorous and extensive phenotyping performed in The Master@Heart trial will provide insight into the exercise-dose response of prolonged and intense endurance training on the whole cardiovascular system. This will enable clinicians better to gauge the risk of cardiovascular disease in athletes and guide future clinical management.

\section{Author affiliations}

${ }^{1}$ Cardiovascular Sciences, KU Leuven University Hospitals Leuven, Leuven, Flanders, Belgium

${ }^{2}$ Cardiology, KU Leuven University Hospitals Leuven, Leuven, Flanders, Belgium ${ }^{3}$ Movement Sciences, Katholieke Universiteit Leuven, Leuven, Flanders, Belgium ${ }^{4}$ Radiology, KU Leuven University Hospitals Leuven, Leuven, Flanders, Belgium ${ }^{5}$ Radiology, Jessa Ziekenhuis Campus Virga Jesse, Hasselt, Limburg, Belgium ${ }^{6}$ Biostatistics and Statistical Bioinformatics, KU Leuven, Leuven, Flanders, Belgium ${ }^{7}$ Cardiology, University Hospital Antwerp, Edegem, Belgium

${ }^{8}$ Cardiology, St Vincent's Hospital, University of Melbourne, Melbourne, Victoria, Australia

${ }^{9}$ Cardiology, Jessa Ziekenhuis Campus Virga Jesse, Hasselt, Limburg, Belgium

Acknowledgements The authors would like to thank the many staff members at the three sites for helping conduct this study. We would particularly like to thank the clinical research assistants Sofie van Soest, Dorien Vermeulen and Daisy Thijs for their dedication and devoted efforts to include, test and follow-up participants.

Contributors All authors contributed to the design of the Master@Heart trial. RDB, $\mathrm{GC}$ and $\mathrm{AB}$ developed the sample size calculation and the statistical analysis plan. $\mathrm{RDB}, \mathrm{CD}, \mathrm{GC}$ and RW drafted the manuscript. All authors read, gave final approval and agreed to be accountable for all aspects of the work, ensuring integrity.

Funding This study is supported by the Fund for Scientific Research Flanders (FWO), Brussels, Belgium. (Grant number: T003717N).

Competing interests RW is supported as a postdoctoral clinical researcher by the Fund for Scientific Research Flanders (FWO Vlaanderen).

Patient and public involvement Patients and/or the public were not involved in the design, or conduct, or reporting, or dissemination plans of this research.

\section{Patient consent for publication Not required.}

Ethics approval The KU Leuven Biomedical Ethics Committee and the committees of UZ Antwerpen and Jessa Ziekenhuis Hasselt approved the study (reference B322201837094)

Provenance and peer review Not commissioned; externally peer reviewed.

Data availability statement No data are available.

Open access This is an open access article distributed in accordance with the Creative Commons Attribution Non Commercial (CC BY-NC 4.0) license, which permits others to distribute, remix, adapt, build upon this work non-commercially, and license their derivative works on different terms, provided the original work is properly cited, appropriate credit is given, any changes made indicated, and the use is non-commercial. See: http://creativecommons.org/licenses/by-nc/4.0/. 
ORCID iD

Ruben De Bosscher http://orcid.org/0000-0002-3163-4781

\section{REFERENCES}

1 GBD 2017 Causes of Death Collaborators. Global, regional, and national age-sex-specific mortality for 282 causes of death in 195 countries and territories, 1980-2017: a systematic analysis for the global burden of disease study 2017. Lancet 2018;392:1736-88.

2 Whelton SP, Chin A, Xin X, et al. Effect of aerobic exercise on blood pressure: a meta-analysis of randomized, controlled trials. Ann Intern Med 2002;136:493-503.

3 Cornelissen VA, Fagard $\mathrm{RH}$. Effects of endurance training on blood pressure, blood pressure-regulating mechanisms, and cardiovascular risk factors. Hypertension 2005;46:667-75.

4 Kelley GA, Kelley KS, Franklin B. Aerobic exercise and lipids and lipoproteins in patients with cardiovascular disease: a metaanalysis of randomized controlled trials. J Cardiopulm Rehabil 2006;26:131-9. quiz 40-1, discussion 42-4.

5 Thomas DE, Elliott EJ, Naughton GA. Exercise for type 2 diabetes mellitus. Cochrane Database Syst Rev 2006:CD002968.

6 Kwaśniewska M, Kostka T, Jegier A, et al. Regular physical activity and cardiovascular biomarkers in prevention of atherosclerosis in men: a 25-year prospective cohort study. BMC Cardiovasc Disord 2016;16:65.

7 Imran TF, Patel Y, Ellison RC, et al. Walking and calcified atherosclerotic plaque in the coronary arteries: the National heart, lung, and blood Institute family heart study. Arterioscler Thromb Vasc Biol 2016;36:1272-7.

8 Koolhaas CM, Dhana K, Golubic R, et al. Physical activity types and coronary heart disease risk in middle-aged and elderly persons: the Rotterdam study. Am J Epidemiol 2016;183:729-38.

9 Batty GD, Shipley MJ, Kivimaki M, et al. Walking PACE, leisure time physical activity, and resting heart rate in relation to disease-specific mortality in London: 40 years follow-up of the original Whitehall study. An update of our work with Professor Jerry N. Morris (19102009). Ann Epidemiol 2010;20:661-9.

10 LaMonte MJ, Fitzgerald SJ, Levine BD, et al. Coronary artery calcium, exercise tolerance, and CHD events in asymptomatic men. Atherosclerosis 2006;189:157-62.

11 Choi S-Y, Sung J, Park HE, et al. Combined effects of exercise capacity and coronary atherosclerotic burden on all-cause mortality in asymptomatic Koreans. Atherosclerosis 2016;251:396-403.

12 Radford NB, DeFina LF, Leonard D, et al. Cardiorespiratory fitness, coronary artery calcium, and cardiovascular disease events in a cohort of generally healthy middle-age men: results from the Cooper center longitudinal study. Circulation 2018;137:1888-95.

13 Blair SN, Morris JN. Healthy hearts--and the universal benefits of being physically active: physical activity and health. Ann Epidemiol 2009;19:253-6.

14 Andersen LB, Schnohr P, Schroll M, et al. All-cause mortality associated with physical activity during leisure time, work, sports, and cycling to work. Arch Intern Med 2000;160:1621-8.

15 Lepers R, Cattagni T. Do older athletes reach limits in their performance during marathon running? Age 2012;34:773-81.

16 Aagaard P, Sahlén A, Braunschweig F. Performance trends and cardiac biomarkers in a 30-km cross-country race, 1993-2007. Med Sci Sports Exerc 2012;44:894-9.

17 Möhlenkamp S, Lehmann N, Breuckmann F, et al. Running: the risk of coronary events : Prevalence and prognostic relevance of coronary atherosclerosis in marathon runners. Eur Heart $J$ 2008;29:1903-10.

18 Aengevaeren VL, Mosterd A, Braber TL, et al. Relationship between lifelong exercise volume and coronary atherosclerosis in athletes. Circulation 2017;136:138-48.

19 Merghani A, Maestrini V, Rosmini S, et al. Prevalence of subclinical coronary artery disease in masters endurance athletes with a low atherosclerotic risk profile. Circulation 2017;136:126-37.

20 Hou Z-hui, Lu B, Gao Y, et al. Prognostic value of coronary CT angiography and calcium score for major adverse cardiac events in outpatients. JACC Cardiovasc Imaging 2012;5:990-9.

21 DeFina LF, Radford NB, Barlow CE, et al. Association of all-cause and cardiovascular mortality with high levels of physical activity and concurrent coronary artery calcification. JAMA Cardiol 2019:4:174-81.

22 Roberts WO, Schwartz RS, Garberich RF, et al. Fifty men, 3510 Marathons, cardiac risk factors, and coronary artery calcium scores. Med Sci Sports Exerc 2017;49:2369-73.

23 Chiu J-J, Chien S. Effects of disturbed flow on vascular endothelium: pathophysiological basis and clinical perspectives. Physiol Rev 2011;91:327-87.
24 Bouassida A, Latiri I, Bouassida S, et al. Parathyroid hormone and physical exercise: a brief review. J Sports Sci Med 2006;5:367-74.

25 Hagström E, Ahlström T, Ärnlöv J, et al. Parathyroid hormone and calcium are independently associated with subclinical vascular disease in a community-based cohort. Atherosclerosis 2015;238:420-6.

26 Sanchis-Gomar F, Mayolas-Pi C, Garatachea N. Inflammation and coronary artery disease: the exercise paradox. Cytokine 2018;111:371-2.

27 Lin J, DeLuca JR, Lu MT, et al. Extreme endurance exercise and progressive coronary artery disease. J Am Coll Cardiol 2017;70:293-5

28 Mozaffarian D, Furberg CD, Psaty BM, et al. Physical activity and incidence of atrial fibrillation in older adults: the cardiovascular health study. Circulation 2008;118:800-7.

29 Morseth B, Graff-Iversen S, Jacobsen BK, et al. Physical activity, resting heart rate, and atrial fibrillation: the Troms $\varnothing$ study. Eur Heart $J$ 2016;37:2307-13.

30 Aizer A, Gaziano JM, Cook NR, et al. Relation of vigorous exercise to risk of atrial fibrillation. Am J Cardiol 2009;103:1572-7.

31 Drca N, Wolk A, Jensen-Urstad M, et al. Atrial fibrillation is associated with different levels of physical activity levels at different ages in men. Heart 2014;100:1037-42.

32 Calvo N, Ramos P, Montserrat S, et al. Emerging risk factors and the dose-response relationship between physical activity and lone atrial fibrillation: a prospective case-control study. Europace 2016;18:57-63.

33 Abdulla J, Nielsen JR. Is the risk of atrial fibrillation higher in athletes than in the general population? A systematic review and metaanalysis. Europace 2009;11:1156-9.

34 Opondo MA, Aiad N, Cain MA, et al. Does high-intensity endurance training increase the risk of atrial fibrillation? A longitudinal study of left atrial structure and function. Circ Arrhythm Electrophysiol 2018;11:e005598.

35 Wilson M, O'Hanlon R, Prasad S, et al. Diverse patterns of myocardial fibrosis in lifelong, veteran endurance athletes. J Appl Physiol 2011;110:1622-6.

36 Tahir E, Starekova J, Muellerleile K, et al. Myocardial Fibrosis in Competitive Triathletes Detected by Contrast-Enhanced CMR Correlates With Exercise-Induced Hypertension and Competition History. JACC Cardiovasc Imaging 2018;11:1260-70.

37 Bohm P, Schneider G, Linneweber L, et al. Right and left ventricular function and mass in male elite master athletes: a controlled contrast-enhanced cardiovascular magnetic resonance study. Circulation 2016;133:1927-35

38 La Gerche A, Burns AT, Mooney DJ, et al. Exercise-induced right ventricular dysfunction and structural remodelling in endurance athletes. Eur Heart J 2012;33:998-1006.

39 Zorzi A, Perazzolo Marra M, Rigato I, et al. Nonischemic left ventricular scar as a substrate of life-threatening ventricular arrhythmias and sudden cardiac death in competitive athletes. Circ Arrhythm Electrophysiol 2016;9.

40 Finocchiaro G, Papadakis M, Robertus J-L, et al. Etiology of sudden death in sports: insights from a United Kingdom regional registry. $J$ Am Coll Cardiol 2016;67:2108-15.

41 Schnell F, Claessen G, La Gerche A, et al. Subepicardial delayed gadolinium enhancement in asymptomatic athletes: let sleeping dogs lie? Br J Sports Med 2016;50:111-7.

42 Mordi I, Carrick D, Bezerra H, et al. T1 and T2 mapping for early diagnosis of dilated non-ischaemic cardiomyopathy in middle-aged patients and differentiation from normal physiological adaptation. Eur Heart J Cardiovasc Imaging 2016;17:797-803.

43 Małek Łukasz A, Barczuk-Falęcka M, Werys K, et al. Cardiovascular magnetic resonance with parametric mapping in long-term ultramarathon runners. Eur J Radiol 2019;117:89-94.

44 Banks L, Altaha MA, Yan A. Abstract 18915: T1-Mapping derived cardiac extracellular volume is not associated with lifelong vigorous exercise in middle-aged endurance athletes. Circulation2017;136.

45 Pujadas S, Doñate M, Li C-H, et al. Myocardial remodelling and tissue characterisation by cardiovascular magnetic resonance (CMR) in endurance athletes. BMJ Open Sport Exerc Med 2018;4:e000422.

46 Messroghli DR, Moon JC, Ferreira VM, et al. Clinical recommendations for cardiovascular magnetic resonance mapping of $\mathrm{T} 1, \mathrm{~T} 2, \mathrm{~T}^{*}$ and extracellular volume: a consensus statement by the Society for cardiovascular magnetic resonance (SCMR) endorsed by the European association for cardiovascular imaging (EACVI). J Cardiovasc Magn Reson 2017;19:75.

47 Harris PA, Taylor R, Thielke R, et al. Research electronic data capture (REDCap)--a metadata-driven methodology and workflow process for providing translational research informatics support. J Biomed Inform 2009:42:377-81. 
48 Harris PA, Taylor R, Minor BL, et al. The REDCap consortium: building an international community of software platform partners. $J$ Biomed Inform 2019;95:103208.

49 Lloyd-Jones DM, Larson MG, Beiser A, et al. Lifetime risk of developing coronary heart disease. Lancet 1999;353:89-92.

50 Chugh SS, Havmoeller R, Narayanan K, et al. Worldwide epidemiology of atrial fibrillation: a global burden of disease 2010 study. Circulation 2014;129:837-47.

51 Dawes TJW, Corden B, Cotter S, et al. Moderate physical activity in healthy adults is associated with cardiac remodeling. Circ Cardiovasc Imaging 2016;9:e004712.

52 Prescott E, Hippe M, Schnohr P, et al. Smoking and risk of myocardial infarction in women and men: longitudinal population study. BMJ 1998;316:1043-7.

53 Prescott $\mathrm{E}$, Scharling $\mathrm{H}$, Osler $\mathrm{M}$, et al. Importance of light smoking and inhalation habits on risk of myocardial infarction and all cause mortality. A 22 year follow up of 12149 men and women in the Copenhagen City heart study. J Epidemiol Community Health 2002;56:702-6.

54 Ding N, Sang Y, Chen J, et al. Cigarette Smoking, Smoking Cessation, and Long-Term Risk of 3 Major Atherosclerotic Diseases. J Am Coll Cardiol 2019;74:498-507.

55 Emerging Risk Factors Collaboration, Sarwar N, Gao P, et al. Diabetes mellitus, fasting blood glucose concentration, and risk of vascular disease: a collaborative meta-analysis of 102 prospective studies. Lancet 2010;375:2215-22.

56 Neaton JD, Blackburn H, Jacobs D, et al. Serum cholesterol level and mortality findings for men screened in the multiple risk factor intervention trial. multiple risk factor intervention trial Research Group. Arch Intern Med 1992;152:1490-500.

57 Nakazato R, Gransar H, Berman DS, et al. Statins use and coronary artery plaque composition: results from the International multicenter confirm registry. Atherosclerosis 2012;225:148-53.

58 Henein M, Granåsen G, Wiklund U, et al. High dose and long-term statin therapy accelerate coronary artery calcification. Int $J$ Cardiol 2015;184:581-6.

59 Puri R, Nicholls SJ, Shao M, et al. Impact of statins on serial coronary calcification during atheroma progression and regression. $J$ Am Coll Cardiol 2015;65:1273-82.

60 Lewington S, Clarke R, Qizilbash N, et al. Age-specific relevance of usual blood pressure to vascular mortality: a meta-analysis of individual data for one million adults in 61 prospective studies. Lancet 2002;360:1903-13.

61 See R, Abdullah SM, McGuire DK, et al. The association of differing measures of overweight and obesity with prevalent atherosclerosis: the Dallas heart study. J Am Coll Cardiol 2007;50:752-9.

62 Sharma S, Drezner JA, Baggish A, et al. International recommendations for electrocardiographic interpretation in athletes. Eur Heart J 2018;39:1466-80.

63 Vlachopoulos C, Aznaouridis K, Stefanadis C. Prediction of cardiovascular events and all-cause mortality with arterial stiffness: a systematic review and meta-analysis. J Am Coll Cardiol 2010;55:1318-27.

64 Laurent S, Cockcroft J, Van Bortel L, et al. Expert consensus document on arterial stiffness: methodological issues and clinical applications. Eur Heart J 2006;27:2588-605.

65 Lang RM, Badano LP, Mor-Avi V, et al. Recommendations for cardiac chamber quantification by echocardiography in adults: an update from the American Society of echocardiography and the European association of cardiovascular imaging. Eur Heart $J$ Cardiovasc Imaging 2015;16:233-71.
66 Nagueh SF, Smiseth OA, Appleton CP, et al. Recommendations for the evaluation of left ventricular diastolic function by echocardiography: an update from the American Society of echocardiography and the European association of cardiovascular imaging. Eur Heart J Cardiovasc Imaging 2016;17:1321-60.

67 Aboyans V, Ricco J-B, Bartelink M-LEL, et al. 2017 ESC Guidelines on the Diagnosis and Treatment of Peripheral Arterial Diseases, in collaboration with the European Society for Vascular Surgery (ESVS): Document covering atherosclerotic disease of extracranial carotid and vertebral, mesenteric, renal, upper and lower extremity arteriesEndorsed by: the European Stroke Organization (ESO) The Task Force for the Diagnosis and Treatment of Peripheral Arterial Diseases of the European Society of Cardiology (ESC) and of the European Society for Vascular Surgery (ESVS). Eur Heart $J$ 2018;39:763-816.

68 Sprynger M, Rigo F, Moonen M, et al. Focus on echovascular imaging assessment of arterial disease: complement to the ESC guidelines (PARTIM 1) in collaboration with the Working group on aorta and peripheral vascular diseases. Eur Heart J Cardiovasc Imaging 2018;19:1195-221.

69 Leipsic J, Abbara S, Achenbach S, et al. SCCT guidelines for the interpretation and reporting of coronary CT angiography: a report of the Society of cardiovascular computed tomography guidelines Committee. J Cardiovasc Comput Tomogr 2014:8:342-58.

70 Cury RC, Abbara S, Achenbach S, et al. Coronary Artery Disease - Reporting and Data System (CAD-RADS): An Expert Consensus Document of SCCT, ACR and NASCI: Endorsed by the ACC. JACC Cardiovasc Imaging 2016;9:1099-113.

71 Abbara S, Blanke P, Maroules CD, et al. SCCT guidelines for the performance and acquisition of coronary computed tomographic angiography: a report of the Society of cardiovascular computed tomography guidelines Committee: endorsed by the North American Society for cardiovascular imaging (NASCI). $J$ Cardiovasc Comput Tomogr 2016;10:435-49.

72 Tilborghs S, Dresselaers T, Claus P, et al. Robust motion correction for cardiac T1 and ECV mapping using a T1 relaxation model approach. Med Image Anal 2019;52:212-27.

73 McDiarmid AK, Swoboda PP, Erhayiem B, et al. Athletic cardiac adaptation in males is a consequence of elevated myocyte mass. Circ Cardiovasc Imaging 2016;9:e003579.

74 La Gerche A, Claessen G, Van de Bruaene A, et al. Cardiac MRI: a new gold standard for ventricular volume quantification during highintensity exercise. Circ Cardiovasc Imaging 2013;6:329-38.

75 Claessen G, Schnell F, Bogaert J, et al. Exercise cardiac magnetic resonance to differentiate athlete's heart from structural heart disease. Eur Heart J Cardiovasc Imaging 2018;19:1062-70.

76 Novillo F, Van Eyndhoven S, Moeyersons J, et al. Unsupervised respiratory signal extraction from ungated cardiac magnetic resonance imaging at rest and during exercise. Phys Med Biol 2019;64:065001.

77 Lancellotti P, Pellikka PA, Budts W, et al. The clinical use of stress echocardiography in non-ischaemic heart disease: recommendations from the European association of cardiovascular imaging and the American Society of echocardiography. Eur Heart $J$ Cardiovasc Imaging 2016;17:1191-229.

78 Karaoğuz MR, Yurtseven E, Aslan G, et al. The quality of ECG data acquisition, and diagnostic performance of a novel adhesive patch for ambulatory cardiac rhythm monitoring in arrhythmia detection. $J$ Electrocardiol 2019;54:28-35. 